\title{
Preparation of Porous Chitosan-Siloxane Hybrids Coated with Hydroxyapatite Particles
}

\author{
Yuki Shirosaki, ${ }^{1}$ Kohei Okamoto, ${ }^{2}$ Satoshi Hayakawa, ${ }^{2}$ Akiyoshi Osaka, ${ }^{2}$ and Takuji Asano ${ }^{3}$ \\ ${ }^{1}$ Frontier Research Academy for Young Researchers, Kyushu Institute of Technology, 2-4 Hibikino, Wakamatsu-ku, \\ Kitakyushu 808-0196, Japan \\ ${ }^{2}$ Graduate School of Natural Science and Technology, Okayama University, 3-1-1 Tsushima-naka, Kita-ku, Okayama 700-8530, Japan \\ ${ }^{3}$ Nikkiso Co., Ltd., Ebisu, Shibuya-ku, Tokyo 150-6022, Japan
}

Correspondence should be addressed to Yuki Shirosaki; yukis@lsse.kyutech.ac.jp

Received 23 July 2014; Accepted 13 October 2014

Academic Editor: Paola Laurienzo

Copyright (C) 2015 Yuki Shirosaki et al. This is an open access article distributed under the Creative Commons Attribution License, which permits unrestricted use, distribution, and reproduction in any medium, provided the original work is properly cited.

This paper describes the apatite deposition of chitosan-silicate porous hybrids derived from chitosan and $\gamma$-glycidoxypropyltrimethoxysilane (GPTMS) in alkaline phosphate solution. The preparation of porous hybrids with needle-like apatite on their surfaces is described. Following apatite deposition the porous hybrids maintained high porosity. The enzymatic degradation rate was low even after 6 months and the porous hybrids were very flexible and cut easily using surgical scissors.

\section{Introduction}

A craniotomy is used to access the brain and can be performed on patients suffering from neurological diseases, traumatic brain injury, brain tumors, hematomas, aneurysms, or a fractured skull. A "burr holes" or "keyhole" is made to allow insertion of a surgical tool, such as a shunt, a drain, an intracranial pressure monitor, or an endoscope or can be used to repair a skull fracture (Figure 1) [1-3].

Following surgery, a "burr hole" must be closed; however current methods, such as hydroxyapatite button $[4,5]$, titanium plates $[6,7]$, or bone substitutes, such as calcium phosphate cements (CPCs), have associated risks [8-12]. CPCs may leak into the brain and make contact with cerebrospinal fluid or blood. Many of them also degrade slowly in the body and may cause delayed inflammation [13]. Hydroxyapatite buttons have similar risks and are not flexible so do not complement and therefore "fit" defects on the skull. Furthermore, titanium plates cause thinning of the surrounding soft tissue and extrusion $[14,15]$ and block observations with MRI. Therefore, new bone substitute materials are required for "burr holes."

Chitosan- $\gamma$-glycidoxypropyltrimethoxysilane (GPTMS) hybrid membranes are cytocompatible with the human osteosarcoma cell, MG63, and human osteoblast (HOB) bone marrow cells [16-19]. In the latter case, a fibrillar extracellular matrix with numerous calcium phosphate globules forms [17]. Apatite deposition on the membranes can be improved by releasing calcium ions from the hybrid matrix. The porous scaffold derived from these hybrid membranes is also cytocompatible and apatite can be deposited in the pores [19]. Our group has shown that the rate of biodegradation is low, even in enzymatic solution [20] and such hybrids would remain until the skull has regenerated.

Chitosan-siloxane hybrids must be washed with $\mathrm{NaOH}$ solution to neutralize residual acetic acid present in the matrix and it is difficult to control the amount of calcium ions present. Huang et al. [21] soaked calcium-containing borate glass spheres in alkaline phosphate solutions to form the apatite. Hayakawa et al. [22] also used alkaline phosphate solution to fabricate hydroxyapatite nanorods on the silicate glass surface. The chitosan-silicate hybrids also contained calcium ions and silicate networks, and apatite formation would be expected in alkaline phosphate solution following neutralizing.

In this study, the chitosan-silicate porous hybrids were soaked in $\mathrm{Na}_{2} \mathrm{HPO}_{4}$ solution and apatite formation was analyzed. The apatite formation was confirmed 


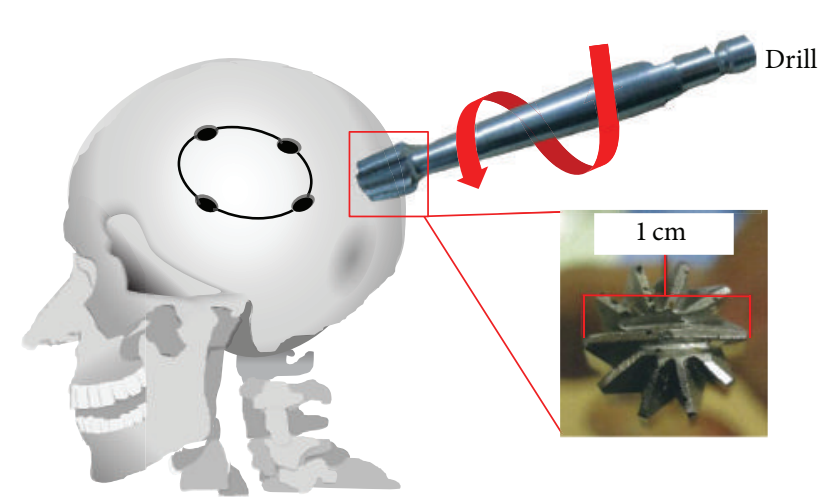

FIGURE 1: Illustration of craniofacial surgery and use of a drill for the initial hole.

TABLE 1: Starting composition of the hybrids (molar ratio).

\begin{tabular}{lccc}
\hline Sample & Chitosan & GPTMS & $\mathrm{CaCl}_{2}$ \\
\hline ChG & 1.0 & 0.5 & 0 \\
ChGCa & 1.0 & 0.5 & 1.0 \\
\hline
\end{tabular}

and the hybrids were characterized with or without surface apatite deposits.

\section{Materials and Methods}

2.1. Preparation of the Porous Hybrids. Chitosan $(0.5 \mathrm{~g}$, high molecular weight, deacetylation: $79.0 \%$, Aldrich, USA) was dissolved in aqueous acetic acid $(0.25 \mathrm{M}, 25 \mathrm{~mL})$. GPTMS (Lancaster, Lancashire, UK) and calcium chloride (Nacalai Tesque, Kyoto, Japan) were added to the chitosan solution to give a molar ratio of chitosan: GPTMS (ChG) $(1: 0.5)$ or chitosan: GPTMS : $\mathrm{CaCl}_{2}$ (ChGCa) $(1.0: 0.5: 1.0)$ as shown in Table 1. One mole of chitosan equates one mole of deacetylated amino groups. The mixtures were stirred for $1 \mathrm{~h}$ at room temperature and a fraction of each resultant sol was poured into a polystyrene container and frozen at $-20^{\circ} \mathrm{C}$ for $24 \mathrm{~h}$. The frozen hybrids were then transferred to a freeze-dryer (FDU-506, EYELA, Tokyo, Japan) for $12 \mathrm{~h}$ until dry. The resultant porous ChG and ChGCa hybrids were then washed with $\mathrm{NaOH}(0.25 \mathrm{M})$ and distilled water to neutralize remaining acetic acid and were again lyophilized. Some ChGCa hybrids were not washed with $\mathrm{NaOH}$ and they were soaked in aqueous $\mathrm{Na}_{2} \mathrm{HPO}_{4}(0.01 \mathrm{M}, \mathrm{pH} 8.8)$ at $80^{\circ} \mathrm{C}$ for various fixed periods (ChGCa $x$ d $[x=$ soaking time]). The hybrids were then washed with distilled water and again lyophilized. Each sample has $2 \mathrm{~mm}$ thickness and $18 \mathrm{~mm}$ in diameter.

\subsection{Structural Characterization and In Vitro Biodegradability} of the Porous Hybrids. The crystal deposits on the sample surfaces were identified with an X-ray diffractometer equipped with a thin-film attachment (TF-XRD, X'pert-PROMPD, PANalytical, Almelo, Netherlands; $\mathrm{CuK} \alpha, \lambda=1.5418 \AA$, $45 \mathrm{kV}$ and $40 \mathrm{~mA}$ ). The incident beam was fixed at $\theta=1^{\circ}$ and the detector was step-scanned around the $2 \theta$ axis from $20^{\circ}$ to $40^{\circ}$ at a rate of $0.02^{\circ} /$ step with a count time of $2 \mathrm{~s}$. The morphology or surface microstructure of the porous hybrids was observed using a field emission scanning electron microscope (FE-SEM, S-4800, Hitachi High-Technology, Tokyo, Japan). Outlines of the pore were traced and the maximum diameter was derived using image analysis software (Image J, National Institutes of Health, Bethesda, MD, USA). At least 20 pores were selected from three different areas of each sample. Bulk density was derived from the thickness of the actual and apparent density and the apparent porosity of the hybrids. The porosity was calculated using (1) [23]

Porosity (\%)

$$
=\frac{\left(V_{m}-V_{p}\right)}{V_{m}} \times 100=\frac{\left[D \times A-\left(m_{m} / r_{p}\right)\right]}{(D \times A)} \times 100,
$$

where $V_{m}$ is the total sample volume, $V_{p}$ is the polymer volume, $r_{p}$ is the density of chitosan $\left(0.858 \mathrm{~g} / \mathrm{cm}^{3}\right), A$ is the proportion of the sample, $m_{m}$ is the weight, and $D$ is the thickness. The degree of cross-linking was evaluated with a ninhydrin assay and defined as the percentage of free amino groups in the hybrids. Pulverized hybrids were suspended in aqueous acetic acid $(0.25 \mathrm{M})$ for $1 \mathrm{~h}$ at room temperature. Ninhydrin solution (ninhydrin reagent L-8500 Set, Wako Chemicals, Osaka, Japan) was added to the suspension and kept at $80^{\circ} \mathrm{C}$ for $20 \mathrm{~min}$. The optical absorbance of the supernatant was then recorded at $568 \mathrm{~nm}$ with an ultraviolet-visible spectrophotometer (UV-2500, Shimadzu Corp., Kyoto, Japan), from which the percentage of free amino groups in the sample was derived [24]. The degree of cross-linking was calculated using

$$
\begin{aligned}
& \text { Degree of cross-linking } \\
& \qquad=\left(1-\frac{A_{568} \text { of each samples }}{A_{568} \text { of chitosan }}\right) \times 100 .
\end{aligned}
$$

The porous hybrids were soaked in phosphate-buffered saline solution (PBS, pH 7.4) to determine water uptake, that is, the amount of water adsorbed into the porous hybrids according to (3). $W_{d}$ and $W_{w}$ stand for the weights before and after PBS soaking, respectively. Consider the following:

$$
\text { Water uptake }(\%)=\frac{100\left(W_{w}-W_{d}\right)}{W_{d}} .
$$

To examine in vitro biodegradability, the porous hybrids were soaked in $1 \mathrm{mg} / \mathrm{mL}$ lysozyme solution (PBS, pH 7.4). The lysozyme solution was changed each 3 days. The weight loss of the porous hybrids was calculated according to (4), where $W_{b}$ and $W_{a}$ stand for the weights before and after being soaked in lysozyme solution, respectively. Consider the following:

$$
\text { Weight loss }(\%)=\frac{100\left(W_{b}-W_{a}\right)}{W_{b}} .
$$

\section{Results and Discussion}

ChG and ChGCa were soaked in $0.01 \mathrm{M} \mathrm{Na}_{2} \mathrm{HPO}_{4}$ for 14 days. And the TF-XRD patterns are shown in Figure 2. Apatite 


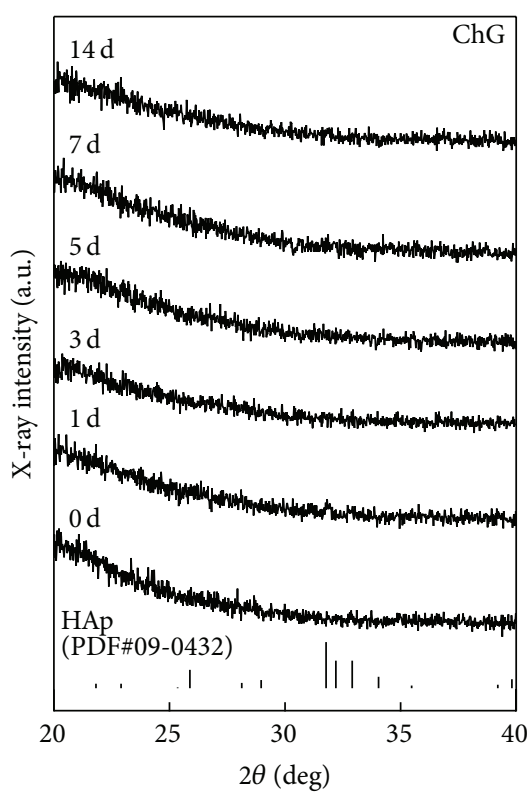

(a)



(b)

FIgURE 2: TF-XRD patterns for ChG and ChGCa before and after soaking in $0.01 \mathrm{M} \mathrm{Na}_{2} \mathrm{HPO}_{4}$ solution for various periods. ${ }^{\circ} \mathrm{HAp}$.

deposits were not detected on ChG but were detected on ChGCa after 1 day. In the case of ChGCa, the pH of the solution decreased from 8.8 to 7.4 because of residual acetic acid used for the preparation of chitosan (sample that was not washed with $\mathrm{NaOH}$ solution before it was soaked in aqueous $\mathrm{Na}_{2} \mathrm{HPO}_{4}$ ). After soaking in $\mathrm{Na}_{2} \mathrm{HPO}_{4}$ solution, the acetic acid and calcium chloride released into the solution and calcium ions reacted with phosphate ions in $\mathrm{Na}_{2} \mathrm{HPO}_{4}$ to form apatite. In this study and in previous work $[18,19]$, EDX analysis indicated the presence of $\mathrm{Si}$ in all the hybrids (ChG, $\mathrm{ChGCa}$, and ChGCa_xd). It means that the silanol groups or siloxane networks exist in the hybrids. The presence of silanol groups on the ChGCa was available to the apatite nuclei [19] and a physiological $\mathrm{pH}$ provides suitable conditions for apatite formation and growth. After 3 days, the apatite growth stopped, indicating that release of calcium ions was immediate but momentary.

Figure 3 shows FE-SEM images of cross-section for the hybrids. In the case of SBF (simulated body fluid, Kokubo solution) [19], spherical deposits covered the surface of ChGCa. In this work, the apatite deposits on the ChGCa in $\mathrm{Na}_{2} \mathrm{HPO}_{4}$ solution were needle-like (d). Apatite also deposited inside of the hybrids regardless of the depth. Calcium ions diffused from the hybrid pore surface and phosphate ions can react only near the interface between the pore surface and solution. Apatite can grow following the diffusion of calcium ions because they are not present in solution and the shape became needle-like.

The chitosan-GPTMS hybrids (both ChGCa and ChG) have interconnected pores and this is consistent with precious reports $[18,19]$. After soaking in $0.01 \mathrm{M} \mathrm{Na}_{2} \mathrm{HPO}_{4}$, the porous microstructure was maintained. Table 2 shows the pore size, porosity, and degree of cross-linking. The pore size for
ChGCa $(69.8 \pm 13.4 \mu \mathrm{m})$ and ChGCa_3 d $(62.1 \pm 12.5 \mu \mathrm{m})$ was slightly higher than that for ChG $(48.8 \pm 16.9 \mu \mathrm{m})$. This is because washing with aqueous $\mathrm{NaOH}$ or soaking in $\mathrm{Na}_{2} \mathrm{HPO}_{4}$ solution causes swelling and an enlargement of the pores. As previously reported [18], ChG and ChGCa have high porosity, with $93.8 \pm 0.2 \%$ and $92.4 \pm 0.4 \%$, respectively. After soaking in $\mathrm{Na}_{2} \mathrm{HPO}_{4}$ solution, this porosity did not change and was $94.8 \pm 0.3 \%$ for ChGCa_3 d. The degree of cross-linking for the porous hybrids was lower than that for solid membrane even with the same composition $[17,18]$. As shown in Table 2, the degree of cross-linking for ChGCa $(28.7 \pm 4.9 \%)$ and ChGCa_3 d $(31.3 \pm 3.9 \%)$ is slightly lower than that for ChG $(36.6 \pm 4.0 \%)$. This is because calcium ions interacted with amino groups in the chitosan matrix [16], so cross-linking is inhibited.

Water uptake in PBS is shown in Figure 4. A plateau or equilibrium is observed for ChGCa and ChGCa_3 $\mathrm{d}$ after $2 \mathrm{~h}$ and after $5 \mathrm{~h}$ for ChG. After soaking in PBS, the shape of the porous hybrids did not change. ChGCa and ChGCa_3 d absorbed 1.5 times more PBS than did ChG. ChGCa has more space for water retention inside the hybrids than does ChG even with apatite deposits on the surface (ChGCa_3 d). On the other hand, ChGCa may release calcium ions into PBS and exchange or react with PBS resulting in high water uptake.

To investigate the biodegradability, the porous hybrids were soaked in lysozyme solution. Table 3 shows the resultant loss in weight. After 1 month, the weight of ChGCa decreased by approximately $50 \%$, while ChG and ChGCa_3 d scarcely changed. This indicates that the release of calcium ions from ChGCa contributes to the weight loss because they do not deposit apatite in lysozyme solution. The ChGCa_3 d scarcely degraded after 1 month and then by approximately $40 \%$ 


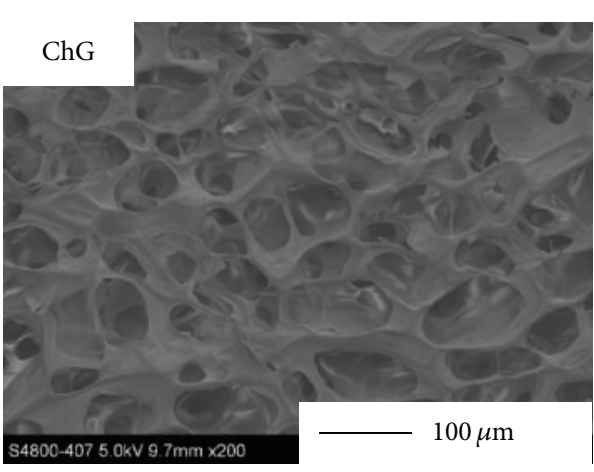

(a)

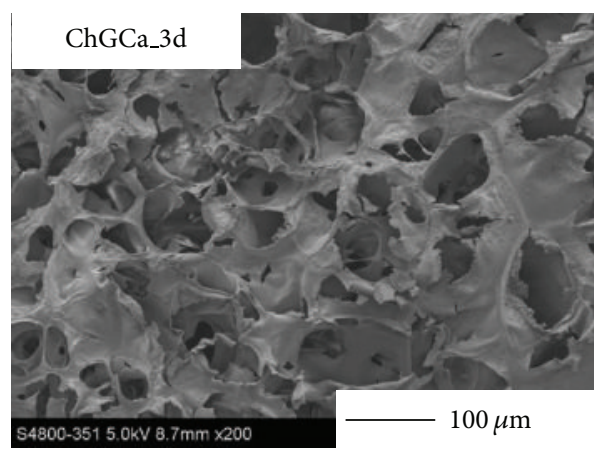

(c)

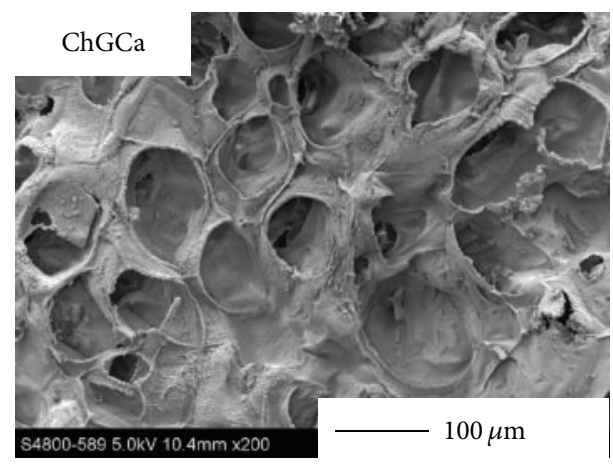

(b)

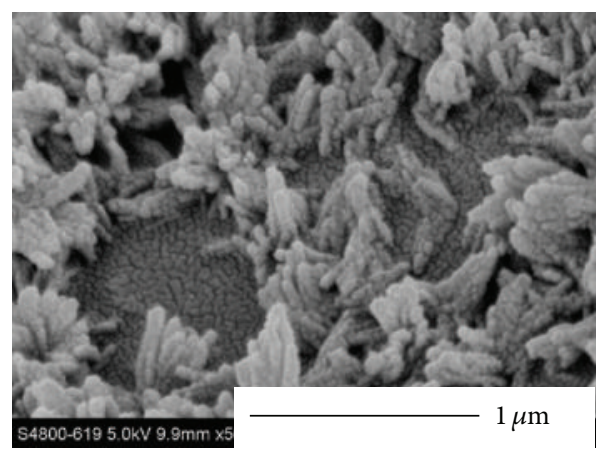

(d)

FIgUre 3: FE-SEM images of cross-section for the ChG, ChGCa, and ChGCa_3 d. The image at lower right is magnification of ChGCa_3 $\mathrm{d}$.

TABle 2: Pore size, porosity, and degree of cross-linking for ChG, ChGCa, and ChGCa_3 d.

\begin{tabular}{|c|c|c|c|}
\hline Sample & Pore size $(\mu \mathrm{m})$ & Porosity (\%) & Degree of cross-linking (\%) \\
\hline $\mathrm{ChG}$ & $48.8 \pm 16.9$ & $93.8 \pm 0.2$ & $36.6 \pm 4.0$ \\
\hline $\mathrm{ChGCa}$ & $69.8 \pm 13.4$ & $92.4 \pm 0.4$ & $28.7 \pm 4.9$ \\
\hline ChGCa_3 d & $62.1 \pm 12.5$ & $94.8 \pm 0.3$ & $31.3 \pm 3.9$ \\
\hline
\end{tabular}

TABle 3: Weight loss (\%) of ChG, ChGCa, and ChGCa_3 d after being soaked in $1 \mathrm{mg} / \mathrm{mL}$ lysozyme/PBS for various periods at $36.5^{\circ} \mathrm{C}$.

\begin{tabular}{lccc}
\hline Sample & \multicolumn{3}{c}{ Soaking period (month) } \\
& 1 & 3 & 6 \\
\hline ChG & $1.01 \pm 1.43$ & $14.87 \pm 5.46$ & $28.11 \pm 2.99$ \\
ChGCa & $49.78 \pm 9.59$ & $56.56 \pm 1.02$ & $65.27 \pm 1.69$ \\
ChGCa_3d & $2.16 \pm 0.75$ & $40.97 \pm 5.92$ & $46.23 \pm 3.92$ \\
\hline
\end{tabular}

within 3 months. Calcium ions interacted with silanol groups derived from GPTMS and the condensation of GPTMS, that is, the formation of a Si-O-Si network, was inhibited by adding calcium ions. The silanol groups in ChGCa were used to deposit the apatite in $\mathrm{Na}_{2} \mathrm{HPO}_{4}$ and the hybrid did not degrade in 1 month in lysozyme solution. However, after a long period of soaking (3-6 months), they started to degrade faster than ChG. ChGCa and ChGCa_3 d absorb more PBS than does $\mathrm{ChG}$ and the enzyme can work in the pores and the matrix. Moreover, ChGCa released calcium ions into lysozyme solution and reduced their weight. Anyway, the enzymatic degradation of a chitosan-silicate hybrid matrix is slow; therefore, this material is suitable for the regeneration of skull.

\section{Conclusion}

The chitosan-GPTMS porous hybrids deposited apatite in alkaline phosphate solution. This means that the calcium ions in the hybrids can be used to form the apatite without loss during washing. The hybrids had high porosity and water uptake property was maintained after apatite deposition. It is expected that the chitosan-GPTMS porous hybrids coated hydroxyapatite can be compatible to the bone defect.

\section{Conflict of Interests}

The authors declare that there is no conflict of interests regarding the publication of this paper. 




Figure 4: Water uptake of ChG, ChGCa, and ChGCa_3d after soaking in PBS for 1 day.

\section{Acknowledgments}

This work was supported in part by the programs in "Improvement of Research Environment for Young Researchers (Kojinsenbatsu)," Ministry of Education Culture, Sports, Science and Technology (MEXT), and the Foundation for the Promotion Ion Engineering.

\section{References}

[1] C. Kilincer, O. Simsek, M. K. Hamamcioglu, T. Hicdonmez, and S. Cobanoglu, "Contralateral subdural effusion after aneurysm surgery and decompressive craniectomy: case report and review of the literature," Clinical Neurology and Neurosurgery, vol. 107, no. 5, pp. 412-416, 2005.

[2] C. G. Bénar and J. Gotman, "Modeling of post-surgical brain and skull defects in the EEG inverse problem with the boundary element method," Clinical Neurophysiology, vol. 113, no. 1, pp. 48-56, 2002.

[3] T. Kawaguchi, S. Fujita, K. Hosoda et al., "Multiple burr-hole operation for adult moyamoya disease," Journal of Neurosurgery, vol. 84, no. 3, pp. 468-476, 1996.

[4] R. Verheggen and H. A. Merten, "Correction of skull defects using hydroxyapatite cement (HAC): evidence derived from animal experiments and clinical experience," Acta Neurochirurgica, vol. 143, no. 9, pp. 919-926, 2001.

[5] D. J. Verret, Y. Ducic, L. Oxford, and J. Smith, "Hydroxyapatite cement in craniofacial reconstruction," Otolaryngology: Head and Neck Surgery, vol. 133, no. 6, pp. 897-899, 2005.

[6] H. Kashimura, K. Ogasawara, Y. Kubo, K. Yoshida, A. Sugawara, and A. Ogawa, "A newly designed hydroxyapatite ceramic burrhole button," Vascular Health and Risk Management, vol. 6, no. 1, pp. 105-108, 2010.

[7] H. V. Easwer, A. Rajeev, H. K. Varma, S. Vijayan, and R. N. Bhattacharya, "Cosmetic and radiological outcome following the use of synthetic hydroxyapatite porous-dense bilayer burrhole buttons," Acta Neurochirurgica, vol. 149, no. 5, pp. 481-485, 2007.

[8] S. Mukherjee, B. Thakur, I. Haq, S. Hettige, and A. J. Martin, "Complications of titanium cranioplasty: a retrospective analysis of 174 patients," Acta Neurochirurgica, vol. 156, no. 5, pp. 989-998, 2014.

[9] J. J. Kuttenberger and N. Hardt, "Long-term results following reconstruction of craniofacial defects with titanium micromesh systems," Journal of Cranio-Maxillofacial Surgery, vol. 29, no. 2, pp. 75-81, 2001.

[10] L. C. Chow, "Next generation calcium phosphate-based biomaterials," Dental Materials Journal, vol. 28, no. 1, pp. 1-10, 2009.

[11] M. J. Provenzano, K. P. Murphy, and L. H. Riley III, "Bone cements: review of their physiochemical and biochemical properties in percutaneous vertebroplasty," American Journal of Neuroradiology, vol. 25, no. 7, pp. 1286-1290, 2004.

[12] J. M. Kuemmerle, A. Oberle, C. Oechslin et al., "Assessment of the suitability of a new brushite calcium phosphate cement for cranioplasty-an experimental study in sheep," Journal of Cranio-Maxillofacial Surgery, vol. 33, no. 1, pp. 37-44, 2005.

[13] A. Moreira-Gonzaiez, I. T. Jackson, T. Miyawaki, K. Barakat, and V. DiNiks, "Clinical outcome in cranioplastry: critical review in long-term follow-up," Journal of Craniofacial Surgery, vol. 14, no. 2, pp. 144-153, 2003.

[14] G. Z. Sanus, T. Tanriverdi, M. O. Ulu, A. M. Kafadar, N. Tanriover, and F. Ozlen, "Use of cortoss as an alternative material in calvarial defects: the first clinical results in cranioplasty," Journal of Craniofacial Surgery, vol. 19, no. 1, pp. 88-95, 2008.

[15] S. H. Yoon, J. S. Burm, W. Y. Yang, and S. Y. Kang, "Vascularized bipedicled pericranial flaps for reconstruction of chronic scalp ulcer occurring after cranioplasty," Archives of Plastic Surgery, vol. 40, no. 4, pp. 341-347, 2013.

[16] Y. Shirosaki, K. Tsuru, S. Hayakawa et al., "In vitro cytocompatibility of MG63 cells on chitosan-organosiloxane hybrid membranes," Biomaterials, vol. 26, no. 5, pp. 485-493, 2005.

[17] Y. Shirosaki, K. Tsuru, S. Hayakawa et al., "Physical, chemical and in vitro biological profile of chitosan hybrid membrane as a function of organosiloxane concentration," Acta Biomaterialia, vol. 5, no. 1, pp. 346-355, 2009.

[18] Y. Shirosaki, T. Okayama, K. Tsuru, S. Hayakawa, and A. Osaka, "Synthesis and cytocompatibility of porous chitosan-silicate hybrids for tissue engineering scaffold application," Chemical Engineering Journal, vol. 137, no. 1, pp. 122-128, 2008.

[19] Y. Shirosaki, T. Okayama, K. Tsuru, S. Hayakawa, and A. Osaka, "In vitro bioactivity and MG63 cytocompatibility of chitosan-silicate hybrids," International Journal of Materials and Chemistry A, vol. 3, no. 3, pp. 1-7, 2013.

[20] Y. Shirosaki, K. Tsuru, S. Hayakawa, and A. Osaka, "Biodegradable chitosan-silicate porous hybrids for drug delivery," Key Engineering Materials, vol. 361-363, pp. 1219-1222, 2008.

[21] W. Huang, D. E. Day, K. Kittiratanapiboon, and M. N. Rahaman, "Kinetics and mechanisms of the conversion of silicate (45S5), borate, and borosilicate glasses to hydroxyapatite in dilute phosphate solutions," Journal of Materials Science: Materials in Medicine, vol. 17, no. 7, pp. 583-596, 2006.

[22] S. Hayakawa, Y. Li, K. Tsuru, A. Osaka, E. Fujii, and K. Kawabata, "Preparation of nanometer-scale rod array of hydroxyapatite crystal," Acta Biomaterialia, vol. 5, no. 6, pp. 2152-2160, 2009. 
[23] H. Hong, J. Wei, and C. Liu, "Development of asymmetric gradational-changed porous chitosan membrane for guided periodontal tissue regeneration," Composites Part B: Engineering, vol. 38, no. 3, pp. 311-316, 2007.

[24] S. Prochazkova, K. M. Vårum, and K. Ostgaard, "Quantitative determination of chitosans by ninhydrin," Carbohydrate Polymers, vol. 38, no. 2, pp. 115-122, 1999. 

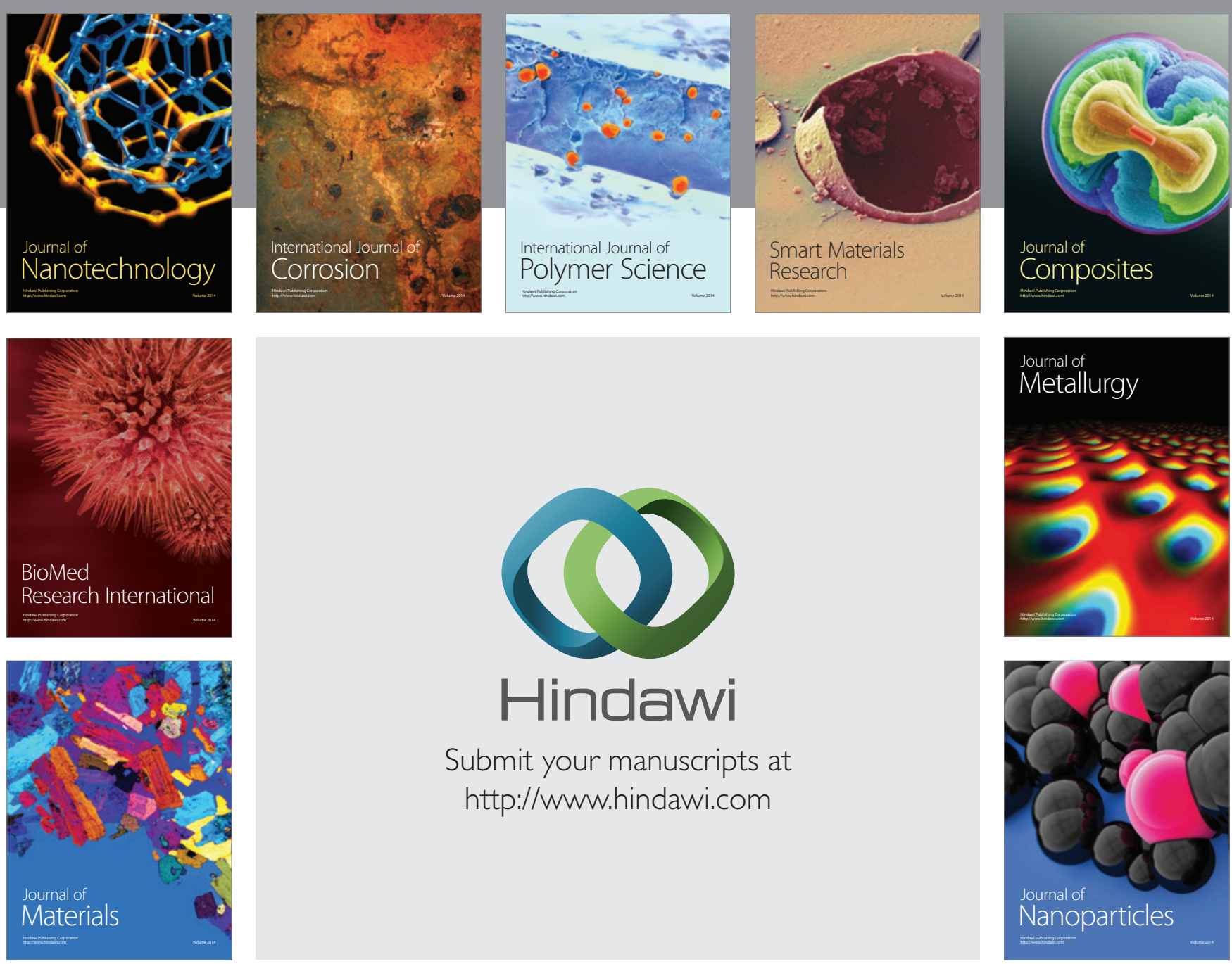

Submit your manuscripts at http://www.hindawi.com
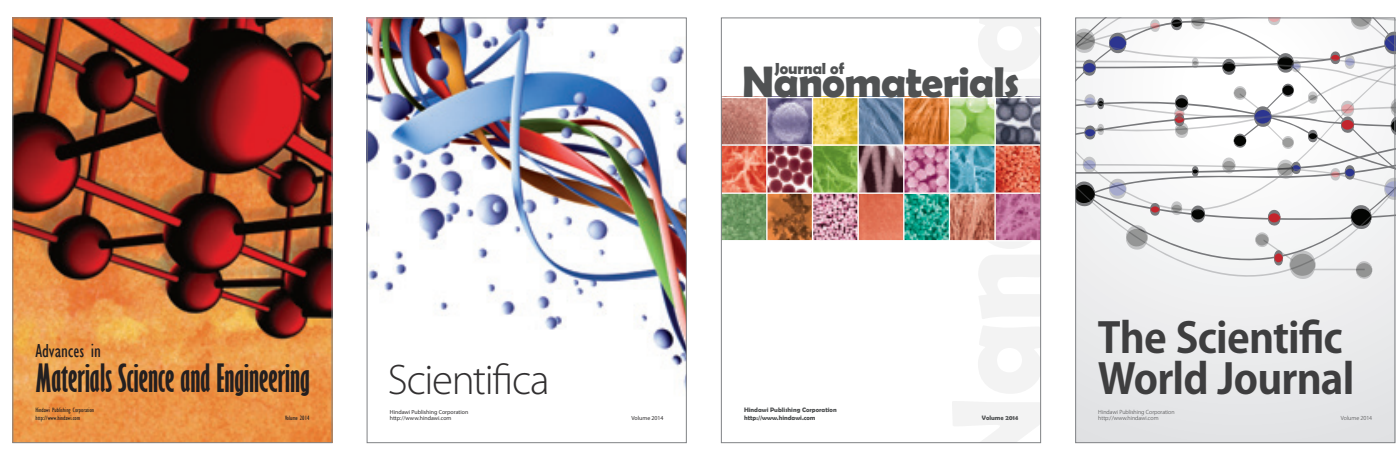

\section{The Scientific World Journal}
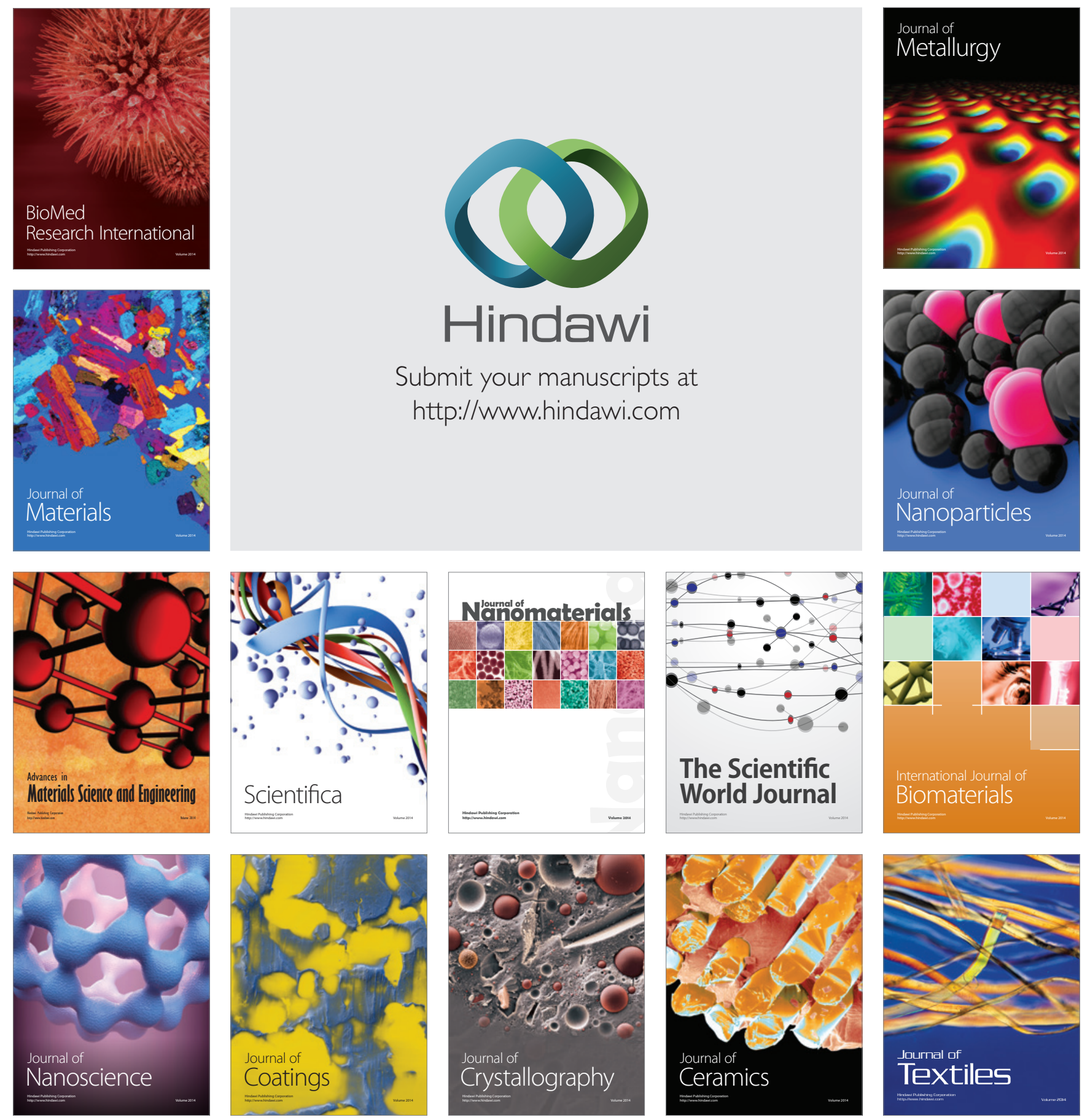